\title{
Corrigendum to "Potential Factors for Psychological Symptoms at Three Months in Patients with Young Ischemic Stroke"
}

\author{
Dongjuan Xu $\mathbb{D}^{1},{ }^{1} \mathrm{Xi}$ Chu, ${ }^{2}$ Kun Wang, ${ }^{3,4}$ Lianyan Wei, ${ }^{1}$ Yunyun Xu, ${ }^{1}$ Xiaomin Huang, ${ }^{5}$ \\ Jinna Li, ${ }^{6}$ Lina Xu, ${ }^{6}$ Lu Yin $\left(D,{ }^{7}\right.$ Hong Liu, ${ }^{8}$ Xiaolei Liu, ${ }^{9}$ Haixia Leng, ${ }^{4}$ Qing Xue, ${ }^{4}$ Mao Peng, \\ Longbin Jia, ${ }^{6}$ and Hongxing Wang ${ }^{4,10,11}$

\footnotetext{
${ }^{1}$ Department of Neurology, Dongyang People's Hospital, Wenzhou Medical University, Zhejiang 322100, China

${ }^{2}$ Health Management Department, Xuanwu Hospital, Capital Medical University, Beijing 100053, China

${ }^{3}$ Department of Neurology, Beijing Puren Hospital, Beijing 100062, China

${ }^{4}$ Department of Neurology, Xuanwu Hospital, Capital Medical University, Beijing 100053, China

${ }^{5}$ Department of Neurology, Ningcheng Center Hospital, Inner Mongolia 024200, China

${ }^{6}$ Department of Neurology, Jincheng People's Hospital, Shanxi 048026, China

${ }^{7}$ Medical Research \& Biometrics Centre, National Centre for Cardiovascular Diseases, Fuwai Hospital, Peking Union Medical College \& Chinese Academy of Medical Sciences, Beijing, China Beijing, 102300, China

${ }^{8}$ Department of Neurology, Heping Hospital Affiliated to Changzhi Medical College, Shanxi 046000, China

${ }^{9}$ Department of Neurology, The First Affiliated Hospital of Kunming Medical University, Yunnan 650032, China

${ }^{10}$ Beijing Psychosomatic Disease Consultation Center, Xuanwu Hospital, Capital Medical University, Beijing 100053, China

${ }^{11}$ Institute of Sleep and Consciousness Disorders, Beijing Institute for Brain Disorders, Capital Medical University,
} \\ Beijing 100053, China
}

Correspondence should be addressed to Hongxing Wang; wanghongxing@xwh.ccmu.edu.cn

Received 24 May 2021; Accepted 24 May 2021; Published 7 June 2021

Copyright (C) 2021 Dongjuan Xu et al. This is an open access article distributed under the Creative Commons Attribution License, which permits unrestricted use, distribution, and reproduction in any medium, provided the original work is properly cited.

In the article titled "Potential Factors for Psychological Symptoms at Three Months in Patients with Young Ischemic Stroke" [1], the authors identified two errors in Table 3 where

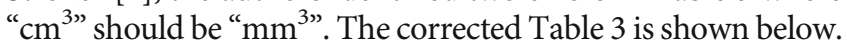


TABLE 3

\begin{tabular}{|c|c|c|}
\hline Variables & OR $(95 \% \mathrm{CI})$ & $P$ \\
\hline \multicolumn{3}{|l|}{ Model for SCL-90-R } \\
\hline Family function (moderate and serious dysfunction vs. good) & $2.50(1.71,3.54)$ & $<0.01$ \\
\hline Hypertension (yes vs. no) & $3.27(1.92,4.27)$ & 0.02 \\
\hline Infarct size ( $\geq 20$ vs. $<20 \mathrm{~mm}^{3}$ ) & $2.39(1.53,3.45)$ & $<0.01$ \\
\hline \multicolumn{3}{|l|}{ Model for depression } \\
\hline Marital status (single vs. married) & $1.23(1.03,1.54)$ & 0.01 \\
\hline Family function (moderate and serious dysfunction vs. good) & $1.21(1.05,1.45)$ & 0.03 \\
\hline Infarct size ( $\geq 20$ vs. $\left.<20 \mathrm{~mm}^{3}\right)$ & $1.74(1.14,3.13)$ & 0.02 \\
\hline \multicolumn{3}{|l|}{ Model for somatization } \\
\hline Family function (moderate and serious dysfunction vs. good) & $2.32(1.51,2.80)$ & $<0.01$ \\
\hline \multicolumn{3}{|l|}{ Model for anxiety } \\
\hline Hypertension (yes vs. no) & $2.41(1.54,3.46)$ & 0.03 \\
\hline
\end{tabular}

Abbreviations: OR: odds ratio; CI: confidence interval; SCL-90-R: Symptom Checklist 90 Revised.

\section{References}

[1] D. Xu, X. Chu, K. Wang et al., "Potential Factors for Psychological Symptoms at Three Months in Patients with Young Ischemic Stroke," BioMed Research International, vol. 2021, Article ID 5545078, 7 pages, 2021. 